\title{
Vitamin B12 Deficiency Resulting in Subacute Combined Degeneration in the Spinal Cord and a Typical Magnetic Resonance Image
}

Tsang-Shan Chen ${ }^{*}$ and Cheng-Yang Hsieh

Department of Neurology, Tainan Sin-Lau Hospital, Tainan, Taiwan

*Corresponding author: Tsang Shan Chen, Department of Neurology, Sin-Lau Hospital, the Presbyterian Church in Taiwan, Tainan, Taiwan 57, sec 1, Dongmen Road, East district, Taiwan City 70142, Taiwan, Tel: +886-919517380; E-mail: tschern@gmail.com

Received date: January 09, 2016; Accepted date: January 22, 2016; Published date: January 29, 2016

Copyright: (C) 2016 Chen TS, et al. This is an open-access article distributed under the terms of the Creative Commons Attribution License, which permits unrestricted use, distribution, and reproduction in any medium, provided the original author and source are credited.

Keywords: Macrocytic anemia; Vitamin B12 deficiency; Subacute combined degeneration

\section{Case Report}

A 78-year-old woman presented with slowly progressive weakness of her hands and numbness in her feet when she stood up or walked. A neurological examination showed generally brisk deep tendon reflexes. Her ability to feel vibrations was reduced, particularly in her lower limbs. She is not a vegetarian and has no history of gastric surgery. Laboratory blood analyses disclosed macrocytic anemia (Hemoglobin [Hb]: $6.4 \mathrm{~g} / \mathrm{dl}$; mean corpuscular volume [MCV]: $137 \mathrm{fl}$ ), and low serum vitamin B12 (110 pg/ml). After a blood transfusion, her Hb rose to $11 \mathrm{~g} / \mathrm{dl}$ and her MCV to $112.5 \mathrm{fl}$. A sagittal cervical spinal magnetic resonance image $(\mathrm{MRI})$ reveals a linear high-intensity T2-weighted lesion in the posterior cord from the C2 to the C5 level (Figure 1: left side, arrows). An axial view shows a high signal intensity T2-weighted linear lesion with an inverted $\mathrm{V}$ shape in the posterior column (Figure: right side, arrowheads).

A vitamin B12 deficiency can result in subacute combined degeneration (SCD), which encompasses pyramidal tracts and posterior column dysfunction. The histology of SCD includes demyelination, vacuolization, and axonal degeneration, typically in the dorsal or lateral spinal cord, but also sometimes seen in the ventral column [1]. MRI features of this type of myelopathy show a linear pattern of increased T2-weighted signal in the posterior column of the cervical cord and an inverted $\mathrm{V}$ sign in cross-sectional images. A nodular pattern of T2-hyperintensity is usually seen in the thoracic cord [2]. An MRI of SCD should be differentiated from an MRI of multiple sclerosis, transverse myelitis, posterior spinal artery syndrome, and HIV-related vacuolar myelopathy.

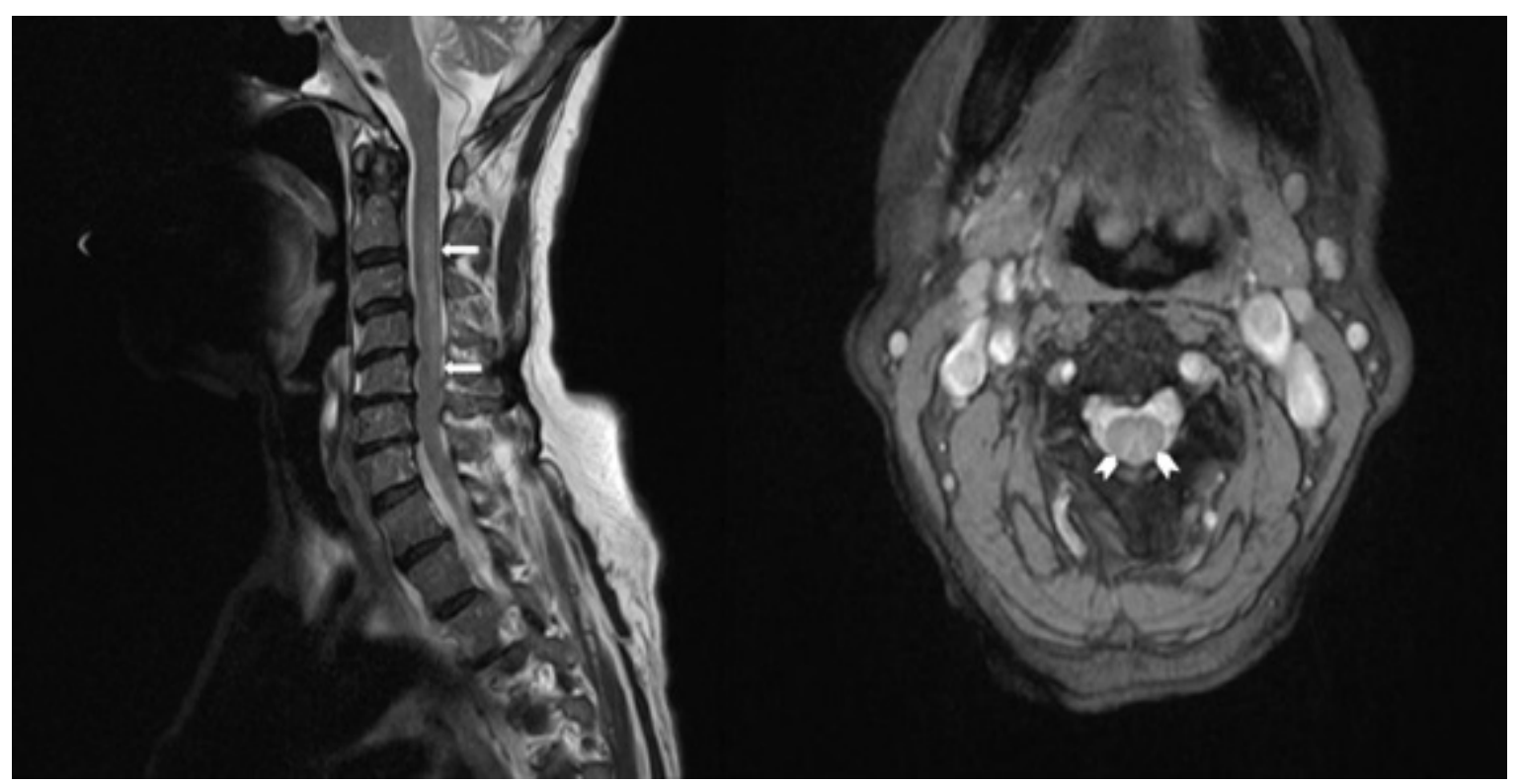

Figure 1: MRI shows a linear high-intensity T2-weighted lesion in the posterior cord from the C2 to the C5 level (left side, arrows) and an axial view shows a high signal intensity T2-weighted linear lesion with an inverted V shape in the posterior column (right side, arrowheads).

\section{References}

1. Timms SR, Curé JK, Kurent JE (1993) Subacute combined degeneration of the spinal cord: MR findings. AJNR Am J Neuroradiol 14: 1224-1227.
2. Sun HY, Lee JW, Park KS, Wi JY, Kang HS (2014) Spinal MR imaging features of subacute combined degeneration patients. Eur Spine J 23: 1052-1058. 